\title{
REFLEXIONES EN TORNO AL DERECHO DE ACCESO A LA INFORMACIÓN DE LOS PARTIDOS POLÍTICOS EN EL PERÚ
}

\section{REFLECTIONS ON THE RIGHT OF ACCESS TO INFORMATION OF POLITICAL PARTIES IN PERU}

\author{
José David Cotrina Campos ${ }^{1}$ \\ Politólogo \\ Universidad Nacional Mayor de San Marcos \\ jose.david.cotrina@gmail.com
}

Perú

\section{SUMARIO}

- Introducción

- El derecho de acceso a la información

- El derecho de acceso a la información en el Perú

- Acceso a la información privada

- ¿Es posible ejercer este derecho en los partidos políticos?

- México como punto de referencia

- Conclusiones

\section{RESUMEN}

Elobjetivo de este estudio es explorar los aspectos normativos e institucionales del derecho de acceso a la información de los partidos políticos en el Perú. Para esto se ha realizado una revisión documental de los aspectos jurídicos, doctrinarios y teóricos de las implicancias de esta norma. Con base en ello, se sostiene que la regulación actual de este derecho es incipiente, pese a que los partidos son entidades cuasi públicas, con responsabilidad e interés público. No obstante, el régimen formal y constitucional que lo regula es limitado; inclusive, el concepto mismo es incipiente en el sistema de partidos. Por otra parte, es probable que gran parte de la argumentación contraria a la extensión de este derecho se deba a que dicha información solicitada se vea confrontada con la protección de la privacidad. De esta manera, mucha de la información relevante que pueda ser sensible al ordenamiento democrático sigue sin conocerse. Por último, se recomienda nivelar este derecho fundamental mediante la legislación gradual de normas para que los partidos brinden información solicitada, y la ampliación del enfoque a partir de la jurisprudencia y evolución institucional desde la perspectiva comparada.

\begin{abstract}
The objective of this study is to explore the normative and institutional aspects of the right of access to information of political parties in Peru. To this end, a documentary review has been made of the legal, doctrinal and theoretical aspects of the implications of this norm. Based on this, it is argued that the current regulation of this right is incipient, although the parties are quasi-public entities, with responsibility and public interest. However, the formal and constitutional regime that regulates it is limited; even the concept itself is incipient in the party system. On the other hand, it is probable that a large part of the argument contrary to the extension of this right is due to the fact that said requested information is confronted with the protection of privacy. In this way, much of the relevant information that may be sensitive to democratic order remains unknown. Finally, it is recommended that this fundamental right be leveled by gradually legislating rules for parties to provide requested information and broadening the approach based on jurisprudence and institutional evolution from a comparative perspective.
\end{abstract}

1 Politólogo por la Universidad Nacional Mayor de San Marcos y egresado de la Maestría en Gobernabilidad del Instituto de Gobierno y Gestión Pública de la Universidad de San Martín de Porres, Perú 


\section{PALABRAS CLAVE}

Derecho al acceso a la información, garantías y recursos constitucionales, partidos políticos, reforma de los partidos políticos, transparencia, orden democrático

\section{KEYWORDS}

Right of access to information, guarantees and constitutional resources, political parties, reform of political parties, transparency, democratic order

\section{INTRODUCCIÓN}

Después de diez años de autoritarismo electoral, cuando los partidos políticos fueron marginalizados, el Perú experimentó una política más competitiva y pluralista; sin embargo, no ha habido un resurgimiento sostenido de los partidos organizados ni mucho menos canales de transparencia y acceso a la información (Levitt y Kostandinova, 2014, p. 526). Las organizaciones políticas tienen bajos niveles de institucionalización, carecen de una programación política coherente y de cuadros técnicos para asumir cargos públicos. Estas debilidades repercuten directamente en el Estado para el diseño e implementación de políticas públicas que favorezcan a las mayorías, manteniendo intereses particulares «a raya» (Tanaka y Vera, 2010, p. 225).

En términos de percepción ciudadana, las organizaciones políticas gozan de una escaza confianza ciudadana: un $47.8 \%$ de peruanos desconfía rotundamente del rol de los partidos políticos (Latinobarómetro, 2016, p. 5)². Estos resultados no son gratuitos: se deben a ciertas prácticas y características impropias a la convivencia democrática, como la personalización de la política, la aparente verticalidad en la forma en que se toman las decisiones internas del partido, así como las constantes denuncias que responden a intereses particulares e inferior capacidad representativa, que las han desestimado como actores indispensables del sistema político, poniendo en duda su capacidad movilizadora y de representación (Alcántara, 2001, p. 4).

2 El secretismo. Una actitud política, vinculada con la herencia autoritaria, justificada por "razones de Estado", lo que afectó seriamente la transparencia fundamental para generar confianza en la ciudadanía, respecto al comportamiento de las autoridades (Miró Quesada, 2017, p. 114).
Como ha sugerido Tuesta Soldevilla (2012), para solucionar este problema de larga data, es indispensable reformar el diseño deficiente de las normas electorales vigentes reguladas por la Constitución, tales como la Ley de Organizaciones Políticas y la Ley Orgánica de Elecciones, entre otras normas.

El debate acerca de la reforma de los partidos o del «sistema de partidos» es amplio y complejo, por lo que tomaremos como punto de partida su vinculación con el derecho de acceso a la información. No obstante, haciendo una evaluación rápida sobre la problemática planteada, en el Perú poco o casi nada se ha hecho para impulsar una reforma de modernización de los partidos políticos desde un enfoque de transparencia y acceso a la información.

$\mathrm{Si}$ bien existen instituciones - tales como el Jurado Nacional de Elecciones (JNE) y la Oficina Nacional de Procesos Electorales (ONPE) - que indirectamente refieren mecanismos de transparencia y rendición de cuentas a la ciudadanía sobre información partidaria, cargos y candidatos de partido, o sobre su situación económico-financiera, entre otros, se observa que incluso estos mecanismos no son los suficientemente eficaces con respecto a la información que brindan. A pesar de que la Ley de Organizaciones Políticas ha dotado a la ONPE de funciones sobre verificación y control de los recursos económicos comprometidos durante la campaña electoral, adolece de sanciones efectivas y oportunas. Es más, ha impuesto sanciones económicas que hasta el momento no han podido ser ejecutadas (Tuesta Soldevilla, 2012a, pp. 28-29), en concreto, la financiación de los partidos, una de las principales sospechas de corrupción para los peruanos en general.

El objetivo de este estudio es explorar los aspectos normativos e institucionales del derecho de acceso a la información de los partidos políticos en el Perú. Para ello se ha realizado una revisión documental de los aspectos jurídicos, así como doctrinarios y teóricos de las implicancias de esta norma. La justificación es simple pero concreta: estas agrupaciones ejercen una responsabilidad pública democrática para el mantenimiento del Estado de derecho. Por ende, la promoción de iniciativa política o legislativa sobre esta materia no debe estar condicionada por una 
cultura de secretismo o "razones de Estado»»» (Miró Quesada, 2017, p. 114); por el contrario, debería convertirse en una herramienta eficiente en la creación de confianza, involucramiento ciudadano y fortalecimiento de la democracia.

\section{EL DERECHO DE ACCESO A LA INFORMACIÓN}

\section{Definición}

Para establecer una definición certera sobre el tema tratado, es indispensable explicar la conexión del derecho de acceso con la transparencia. Parada Vásquez $(2015$, p. 226) concibe la transparencia como el principio por el que se debe gestionar públicamente, ya sea a la vista o al escrutinio público. Entonces, dicha conexión con el acceso reside en una dimensión objetiva: el derecho de acceso a los documentos administrativos. Asimismo, como indica Mateo Maciá (2003), esta conexión tiene una doble vertiente: ofrece mayor facilidad en el acceso a la información, la cual se plasma en la documentación solicitada o proporcionada a fin de generar una mayor inteligibilidad de los procesos en la toma de decisiones Es así que el carácter de este derecho con la transparencia se encuentran casi siempre ligados.

El acceso a la información pública puede entenderse como un derecho fundamental que faculta a la ciudadanía a solicitar al Gobierno y administración determinada información pública no excluida por razones legítimamente tasadas, así como de obtener respuesta en un tiempo razonable (Palomares, 2017, p. 39). Con su aplicación, la ciudadanía obtiene información acerca de la actuación de los gobiernos. Sin lugar a dudas, el derecho de acceso a la información pública salvaguarda otros derechos fundamentales (Santana, 2014).

La relevancia de este derecho tiene características muy propias de sociedades democráticas. En su sentido más amplio, es decir, como derecho a la información, en el artículo 19. ${ }^{\circ}$ de la Declaración Universal de los Derechos Humanos, es entendido como una garantía fundamental que toda persona posee para atraerse información, así como a informar y ser informada. Como indican Precht y Figueroa (2017, p. 2), este derecho resulta ser un prerrequisito indispensable para la reformulación de un nuevo contrato social basado en la gestión de las expectativas de la ciudadanía de forma abierta y visible a los ojos de cualquier persona.

En similar tenor, el vínculo del derecho al libre acceso a la información pública con el robustecimiento de la opinión pública es vital para la democracia. Como señalan Peled y Rabin (2011), la libertad de expresión y el derecho a la información van más allá de la teoría. Las capacidades delosindividuos, grupos deintereses y organizaciones de participar activamente en los asuntos decisivos del debate político, así como la posibilidad de incluir temas en la agenda pública, están sumamente vinculadas a su capacidad de obtener información relevante. Precisamente, los ciudadanos abogan por un gobierno transparente mediante el ejercicio de este derecho.

Para Bobbio (2001), la transparencia, en el ejercicio del Poder público, es un elemento esencial en un sistema democrático, puesto que permite la constante crítica. Así, la publicidad de los actos del Poder público sería la transparencia del ejercicio de todos los actos de los órganos de Gobierno. No obstante, pese a que el tema no es nuevo, parece que todos los días es sencillo encontrar «razones de Estado» en las esferas del Poder para vulnerar el derecho al acceso a la información. Por esa razón, es necesario encontrar nuevos mecanismos que fortalezcan el derecho de acceso a la información pública con la finalidad de ampliar el acceso que se genera en los órganos de Gobierno (Hernández, 2010, p. 20).

En la siguiente sección se esbozarán los principios que rigen el derecho de acceso a la información.

\section{El principio de transparencia}

Se refiere a la apertura y flujo de información de las organizaciones políticas y burocráticas al dominio público (Hernández, 2010, pp. 63- 65). Esto la vuelve accesible a todos los posibles actores interesados, permitiendo su revisión y análisis, así como la detección de posibles anomalías. Incluye cuestiones como la publicación de cuentas y presupuestos gubernamentales auditados, estadísticas financieras, comerciales y monetarias, compensaciones de funcionarios públicos y privados, y datos sobre financiamiento de campañas políticas (Valverde, 2007). 


\section{El principio de publicidad}

Consiste en dar a conocer y poner al alcance de los gobernados - por todos los mecanismos posibles - las razones o criterios que se tuvieron en cuenta para la toma de decisiones de cualquier rango público. Por tanto, cada órgano de Gobierno estaría obligado a brindar la información que generen o tengan en sus manos según su propia naturaleza (Palomares, 2017, p. 261). Toda información de la que disponga el Estado debería ser pública, más la denegación del acceso debe ser excepcional; solo podrá ser fundamentada bajo aquellas razones que estén incluidas en la ley de acceso a la información, tales como la seguridad nacional, protección de datos personales, secretos comerciales, o la prevención o investigación de delitos (Access Info Europe, 2010).

\section{El principio democrático}

Este principio es determinante en muchos aspectos. Va desde la forma que adopta el Estado hasta la legitimidad y organización de los órganos de Gobierno. En su relación con el derecho en mención, pretende que el acceso a los ciudadanos sea lo más completo posible a fin de consolidar el carácter democrático de las instituciones y la confianza pública en la administración (Palomares, 2017, pp. 262263). También es la clave en la legitimidad y organización de los órganos de Gobierno, así como un elemento esencial en el sistema normativo de un país. (Hernández, 2010, p. 60)

\section{E1 principio de participación política}

Es el derecho que tienen todas las personas de formar parte de la vida política de un país o región, así como la discusión de todos los asuntos públicos en los que desean participar. Hay participación política siempre que existe un libre acceso a los documentos oficiales, requisito medible para una mejor transparencia de la administración. Mediante la participación política se espera que la administración pública garantice una mayor eficiencia en la implementación de sus políticas (Palomares, 2017, p. 264).

\section{EL DERECHO DE ACCESO A LA INFORMACIÓN EN EL PERÚ}

\section{El derecho de acceso a la información}

Es un derecho fundamental que en el Perú, a diferencia de otros países, es reconocido por la Constitución Política de 1993. La doctrina nacional ha puesto especial énfasis en el análisis de este respecto, por ejemplo, al genérico derecho de petición. Así, se ha dicho que mediante el derecho contemplado en el numeral 5 del artículo $2 .^{\circ}$, las personas tienen la posibilidad de solicitar y recibir información de cualquier entidad pública, sin expresión de causa. Rubio Correa (1999, p. 219) enfatiza este imperativo señalando que el derecho no es solo a solicitar — para lo cual bastaría el derecho de petición establecido en el numeral 20 del mismo artículo- sino también a recibir información. Solo están obligadas a suministrarla las entidades que ejercen función pública, es decir, las que tienen como finalidad central el servicio a las personas.

En el Perú, gran parte de este tipo de iniciativas en el terreno político ha estado enfocada básicamente a mejorar los mecanismos de transparencia y rendición de cuentas de todos los niveles de Gobierno y a hacer frente a los actos de corrupción y autoritarismo en la década del fujimorismo. No obstante, la principal crítica sería la ausencia de un mecanismo o un órgano de recurso por vía administrativa. En ese sentido, los ciudadanos tienen derecho a la información; sin embargo, si se les niega, tienen que recurrir a los tribunales, lo que implica un coste económico y de tiempo (Palomares, 2017, p. 176).

Con la promulgación de la Ley de Transparencia y Acceso a la Información Pública (Ley N. ${ }^{\circ} 27806$ ), la cual apoya e instrumentaliza la implementación política institucional de la misma, se determinan varias pautas sobre la obligación que tienen las instituciones del Gobierno a brindar información a la ciudadanía, siempre y cuando no sea de carácter confidencial o relacionada a la seguridad nacional. Al respecto, es importante resaltar que el Perú es uno de los países que tiene una de las leyes más antiguas de la región promulgadas desde el 2001.

El Habeas Data (artículo 200. , inciso 3) es una garantía constitucional vinculada al derecho de acceso a la información pública y cuya finalidad es la tutela jurisdiccional del derecho en mención. Para Dalla Vía y Basterra (1999), esta garantía es la representación convencional de hechos, conceptos o instrucciones de forma apropiada para la comunicación y procesamiento por medios automáticos. 
La jurisprudencia del Tribunal Constitucional ha realizado varios aportes relevantes sobre los alcances de este derecho. Con respecto al contenido de la información a entregarse, esta debe ser «oportuna, incondicional y completa» (Tribunal Constitucional, 2007). Inclusive, se ha precisado que la información pública solicitada debe ser entregada en términos mínima o elementalmente razonables, lo que supone que esta deberá ser cierta, completa, clara y, además, actual (Tribunal Constitucional, 2003). Asimismo, han considerado que el derecho de acceso se vería afectado siempre que la información que se proporciona es fragmentaria, desactualizada, incompleta, imprecisa, falsa, no oportuna o errada. En suma, este derecho también ofrece garantías para la entrega de la información en términos de calidad y la celeridad (Tribunal Constitucional, 2002).

Por su parte, el derecho de petición es otro recurso vinculado al derecho de acceso a la información. La Constitución Política (artículo $2 .^{\circ}$, inciso 20) reconoce el derecho de toda persona a formular peticiones por escrito, individual o colectivamente, ante la autoridad competente, la que está obligada a dar al interesado una respuesta también por escrito dentro del plazo legal, bajo responsabilidad. Desde la doctrina jurídica, una definición comprehensiva de este recurso - de acuerdo con Chávez Salazar (1999) - sería el derecho de toda persona que no es titular de derechos subjetivos o de intereses legítimos, de dirigir peticiones a los organismos, órganos y personas-órgano que ejercen las funciones del Poder sobre materias de competencia de estos.

En esencia, la aplicación de este derecho fundamental tiene suma importancia en el ámbito de la participación ciudadana, pues permite a las personas dirigirse a los poderes públicos -es decir, tanto a los órganos parlamentarios como a los gobiernos - con una petición cuyo contenido puede ser diverso. No siempre se acepta el carácter político de este derecho, pues es casi evidente que brinda la posibilidad a la ciudadanía de participar en la actividad del ente estatal.

Por otra parte, y con la finalidad de desarrollar este derecho con mayor amplitud, este recurso se encuentra regulado por la Ley de Procedimiento Administrativo General, básicamente en sus artículos $106 .{ }^{\circ}$ y siguientes, y ofrece hasta cinco ámbitos de operatividad del derecho en mención: 1. La petición gracial, referida a la obtención de una decisión administrativa como consecuencia de la discrecionalidad y libre apreciación de un ente administrativo; 2 . la petición subjetiva, referida a la solicitud individual o colectiva que tiene por objeto el reconocimiento administrativo de un derecho administrativo; 3. la petición cívica, referida a la representación de un grupo indeterminado de personas o de la colectividad en su conjunto, la cual tiene por objeto la protección y promoción del bien común y el interés público; 4. la petición informativa, referida a la obtención de documentación oficial contenida en los bancos informativos o registros manuales de la institución requerida; y 5. la petición consultiva, referida a la obtención de un asesoramiento oficial con relación a una materia administrativa concreta, puntual $\mathrm{y}$ específica (Tribunal Constitucional, 2002).

No obstante, también se identifican ciertas limitaciones de esta norma, pues obliga a la autoridad a recibir la petición, pero no necesariamente puede aprobarla (a veces simplemente la puede denegar). Entonces, no tendría la obligación de satisfacer lo solicitado (Tribunal Constitucional, 2012) o no obtendría una respuesta favorable a lo solicitado (Tribunal Constitucional, 2001).

\section{ACCESO A INFORMACIÓN PRIVADA}

Lo expuesto en la sección anterior corresponde a varios aspectos normativos de alcance gubernamental; sin embargo, ¿el alcance solo debería intervenir en el ejercicio de la función pública y no a entidades privadas, las cuales, en este caso, serían los partidos políticos? Desde la jurisprudencia suministrada, en esta sección se analizará la relación de las entidades privadas con respecto a los instrumentos que forman parte del derecho de acceso a la información.

Al parecer, el legislador ha contemplado a las entidades privadas como sujeto pasivo del derecho de acceso a la información pública, aunque solo se estarían considerando a las que gestionen servicios públicos o ejerzan una función administrativa (Ministerio de Justicia y Derecho Humanos, 2014), tal como se contempla en el artículo $90^{\circ}$ de la Ley N. ${ }^{\circ}$ 27806. Un ejemplo de ello es la jurisprudencia del Tribunal Constitucional 
(2010), que considera factible la solicitud de determinada información a los colegios profesionales por ser entidades autónomas con personalidad de derecho público, así como la de los notarios en la medida en que estarían cumpliendo una función pública (Tribunal Constitucional, 2004).

Por otra parte, el Tribunal ha considerado inicialmente que estas personas jurídicas privadas únicamente tenían el deber de informar sobre sus servicios, tarifas y las funciones administrativas que realizan. No obstante, se ha admitido la entrega de información de diverso orden - por ejemplo, sobre reclamaciones efectuadas, criterios de evaluación para acceder a la universidad e, incluso, grabaciones de programas radiales.

Con base en lo analizado y descrito, los partidos políticos cuentan con cierta normativa que, directa o indirectamente, proveen al ciudadano, en cuanto son - asociaciones de ciudadanos que constituyen personas jurídicas de derecho privado cuyo objeto es participar por medios lícitos, democráticamente, en los asuntos públicos del país - de acuerdo a la Ley de Organizaciones Políticas - y, como indica Sartori (2000), son actores centrales del sistema democrático.

Con respecto a su financiamiento, existe un sistema de control externo que le compete a la Gerencia de Supervisión de Fondos Partidarios (GSFP), oficina adscrita a la ONPE. Asimismo, de acuerdo al artículo 34. - De la Ley Organizaciones, deben presentar un informe financiero a los seis meses de concluido el ejercicio anual; sin embargo, es muy frecuente que dichas organizaciones desestimen presentar de manera cooperativa dicha información, pues carecen de sanciones por incumplimiento.

La misma situación ocurre con la publicidad de la contabilidad. La norma que regula este componente es poco exigente. Obliga a que los partidos lleven libros de contabilidad y que dicha conservación dure hasta diez años después de realizadas las actividades. (Tuesta Soldevilla, 2008, p. 791). Pese a ello, y al no establecerse mecanismos de transparencia en la ley, cualquier actividad relacionada con el acceso se encuentra a discreción de este tipo de organizaciones.
Con la implementación del Reglamento de Financiamiento y Supervisión de Fondos Partidarios - aprobado por la ONPE en enero de 2018 - se espera la reglamentación de las disposiciones legales relativas al financiamiento $\mathrm{y}$ gastos de las organizaciones políticas, candidatos a cargos de elección popular y rendición de cuentas de los promotores y autoridades sometidas a revocación.

Por otra parte, en el Registro de Organizaciones Políticas del JNE solo se cuenta con un aplicativo en el que se observa un registro de los partidos en tiempo real y una relación sobre el padrón de afiliados de los partidos registrados; sin embargo, en su mayor parte está actualizado únicamente hasta octubre de 2017. Por último, con respecto a la información relacionada a los candidatos, se cuenta con el portal Infogob del JNE, el cual permite visualizar los datos de todos los candidatos que postularon por un cargo político, los partidos o movimientos regionales o locales que existen por localidad, así como los resultados de las elecciones. Sin duda, este tipo de información, aunque no es directamente proporcionada por los partidos, es valiosa por la cantidad de información sistematizada que brinda a la ciudadanía.

\section{¿ES POSIBLE EJERCER ESTE DERECHO EN LOS PARTIDOS POLITICOS?}

El derecho de acceso a la información parece o se percibe tácitamente ligado a la democracia, más aún si trasladamos su relevancia en estos tiempos: un gobierno democrático es igual a un gobierno transparente. Pese a ello, señala Aguilar Rivera (2008, p. 9) que esta figura resulta engañosa, pues aún se está lejos de contar con la claridad conceptual que requerimos. Por tanto, se identifica una indeterminación conceptual.

Si bien este derecho es considerado como una herramienta de control del manejo del erario público por parte del Poder Ejecutivo, es posible que deba ampliarse la lista de sujetos pasivos en el ámbito de aplicación subjetivo. Por ejemplo, los primeros de esta lista pueden ser el Poder Judicial y el Parlamento, y, posteriormente, se puede extender el ámbito de aplicación del derecho a los partidos políticos, entre otras entidades privadas, para que se aprecie en su máximo esplendor. 
Como se observó anteriormente, las entidades privadas que prestan servicios públicos están obligadas a una publicidad activa. En el caso de los partidos políticos que no son órganos estatales o titulares de poder público ni ejercen funciones de trascendencia política, sino que son de base asociativa, la información analizada serviría de base para sopesar el argumento de la obligación de publicitar y no impedir o vulnerar el derecho de acceso a la información que requiera un ciudadano. Si bien no están obligados, sería la opción más beneficiosa en aras de mejorar nuestra democracia. El requerimiento de acceso motivaría una nueva adecuación normativa de transparencia propia de la concepción democrática y constitucionalista en este país.

\section{MÉXICO COMO PUNTO DE REFERENCIA}

En México se incorporan ciertos mecanismos legales que permiten a la ciudadanía solicitar información de los partidos políticos en materia electoral. No obstante, pese a que el desarrollo de este derecho es mucho más desarrollado que cualquier país de la región, también presenta defectos propios del desenvolvimiento de los partidos en el sistema político mexicano.

Sus partidos tienen la obligación de presentar informes ante el Instituto Federal Electoral, y la Comisión de Fiscalización de los Recursos Públicos de los Partidos y Agrupaciones Políticas puede ordenar auditorías y verificaciones. Además, los resultados deben ser públicos al concluir el procedimiento de fiscalización respectivo. Pese a todo, la Constitución Mexicana no los obliga a hacer pública información fundamental, lo que resulta contradictorio a la máxime de promover la participación del pueblo en la vida democrática e incorporar a los ciudadanos al ejercicio del poder público.

Cualquier ciudadano de dicho país puede solicitar al Instituto Federal Electoral la información relativa al uso de los recursos públicos que reciban los partidos políticos y las agrupaciones políticas nacionales, pero estos no están obligados a publicar información sobre sus fuentes de financiación. Lo ideal hubiera sido que los partidos políticos hubieran estado obligados no solo a transparentar los resultados de auditorías, verificaciones y resoluciones, sino también el proceso mismo de gestación de tales decisiones (Cárdenas, 2005).

Por otra parte, existen casos en que los partidos políticos se han negado a dar información respecto de sus afiliados o militantes. Para defender su negativa, se escudan en la protección de los datos personales. Por ejemplo, en el 2008, el Tribunal Electoral examinó el caso de la demanda de un ciudadano que solicitó información al Partido de la Revolución Democrática (PDR) para que este le facilitara el padrón de afiliados por Entidad Federativa. Sin embargo, el PRD, cumpliendo con un requerimiento de la Unidad de Enlace del Instituto Federal Electoral, solo presentó un documento en el cual indicaba el número total de afiliados por cada Entidad Federativa bajo el argumento de que estaban protegiendo los datos personales de sus afiliados.

Así, el colegiado electoral determinó que la entrega del padrón de afiliados con los nombres, apellidos y la especificación de la Entidad Federativa a la que pertenecía cada uno no afectaba el principio de confidencialidad del dato personal. Divulgar solo los datos brindados por la Entidad Federativa resultaría complicado e imposible de identificar, por lo que vulneraba el derecho de acceso a la información.

\section{CONCLUSIONES}

Los partidos políticos son entidades cuasi públicas, con responsabilidad y de interés público. No solo son asociaciones altruistas; son organizaciones complejas $\mathrm{y}$, producto de su dinámica, se configura la visión de un país, así como la orientación de las políticas públicas. De esta manera, los beneficios de la divulgación y acceso a la información con la que cuentan superan con creces el derecho a la protección de la privacidad de estas asociaciones.

Es cierto que existe un régimen formal y constitucional que regula el derecho de acceso a la información; sin embargo, aún falta recorrer muchos caminos. El concepto mismo de este derecho es incipiente en el actual sistema de partidos. Las instituciones políticas que la regulan, al parecer, la proporcionan indirectamente, más no han desarrollado mecanismos que permitan a los ciudadanos solicitarla. 
Existe una probabilidad de que el derecho de acceso a la información se vea confrontado con la protección de la privacidad. Debe tenerse en cuenta que aún es una preocupación en el Perú por el pasado autoritario que vivió la democracia. Por ello, es cuestión de ser minucioso con respecto a las formas en las que operaría la norma en los partidos políticos. No obstante, en la espera es probable que mucha de la información relevante que sea sensible a nuestro ordenamiento democrático siga sin conocerse.

Este derecho fundamental puede ser desarrollado o nivelado. Así, se presentan los siguientes puntos a tomar en consideración: la primera es la voluntad política, básicamente de los legisladores, para enfatizar el acceso a través de la creación de una normativa que obligue gradualmente a los partidos a brindar la información solicitada; en segundo lugar, ampliar el enfoque de este derecho a partir de la jurisprudencia y evolución institucional desde la perspectiva comparada. En tal sentido, existe un avance normativo $y$, sobre todo, institucional de otros países, por ejemplo, de México.

\section{FUENTES DE INFORMACIÓN}

\section{Fuentes bibliográficas}

Alcántara Sáez, M. (abril de 2001). Los partidos políticos en América Latina. América Latina Hoy (27), pp. 17-35.

Bobbio, N. (2001). El futuro de la democracia (3. ${ }^{\text {a }}$ ed.). México D.F,: Fondo de Cultura Económica.

Cárdenas, J. (julio de 2005). El acceso a la información de los partidos políticos. Derecho Comparado de la información (6). Instituto de Investigaciones Jurídicas de la UNAM.

Casas-Zamora, K., y Zovatto, D. (2015). El costo de la democracia: apuntos sobre la regulación del financiamiento político en América Latina. Washington D.C: Latina America Initiative Foreign Policy at Brookings.

Dalla Vía, A. y Basterra, M. (1999). Habeas Data y otras garantías constitucionales. Buenos Aires: Nemesis.

Levitt, B. y Kostandinova, T. (2014). Personalist Parties in the Third Wave of Democratization: a comparativa analysis of Perú and Bulgaria. Politics \& Policy, 42(4), pp. 513-547. doi:10.1111/polp.12084
Maciá, M. (2003). El derecho a la información en el ordenamiento jurídico europeo. Barcelona: Ariel.

Miró Quesada Rada, F. (2017). Partidos políticos y calidad de la democracia: el caso peruano. Iuis Inkarri, pp. 113 - 139.

Parada Vásquez, R. (2015). Derecho Administrativo I, Parte General. Madrid: Marcial Pons.

Peled, R. y Rabin, Y. (2011). The Constitutional Right to Information. Columbia Human Rigths Law Review, 42(2), pp. 357-401. Recuperado el 17 de febrero de 2018 de The Constitutional Right to Information

Sartori, G. (2000). ¿Qué es la democria? Madrid: Taurus.

Tanaka, M. y Vera, S. (2010). Perú: la dinámica "neodualista" de una democracia sin sistema de partidos. En Cameron, y Luna, Democracia en la región (pp. 197-242). Lima, Perú: Instituto de Estudios Peruanos. Recuperado el 20 de febrero de 2018

Tribunal Constitucional, 0551-2001-AA/TC (Tribunal Constitucional 2001).

Tribunal Constitucional, 01042 - 2002 -AA/ TC (2002).

Tribunal Constitucional, 01797 -2002 - HD (Tribunal Constitucional 2002).

Tribunal Constitucional, 00007-2003-AI (2003).

Tribunal Constitucional, 0301- 2004 HD/TC (Tribunal Constitucional 5 de marzo de 2004).

Tribunal Constitucional, 04885-2007-HD (2007).

Tribunal Constitucional, 1266 - 2010-HD/ TC (Tribunal Constitucional 27 de agosto de 2010).

Tuesta Soldevilla, F. (2012a). Partidos políticos en el Perú: necesidad de una reforma. En Campos, Democracia, humanismo y poítica. Homenaje a Pedro Planas (pp. 67-94). Lima: Fondo Editorial de la Universidad de Lima. 


\section{Fuentes electrónicas}

Access Info Europe. (2010). El Derecho de Acceso a la Información: definición, protección internacional del derecho y principios básicos. Access Info Europe. Madrid: Access Info Europe. Recuperado el 28 de febrero de 2018 de https://www.access-info.org/wp-content/ uploads/El_Derecho_de_acceso_a_la_ informacin._principios_bsicos.pdf

Aguilar Rivera, J. A. (2008). Transparencia $y$ democracia: claves para un concierto (vol. 10). México D.F., México: Instituto Nacional de Transparencia, Acceso a la Información y Protección de Datos Personales. Recuperado el 20 de febrero de 2018 de http://www.resi.org.mx/ icainew_f/images/Biblioteca/Cuaderno\%20 transparencia/cuadernillo10.pdf

Asociación Civil Trasnparencia. (2016). Informe de observación del proceso electoral. Elecciones generales 2016. Primera vuelta. Lima: Transparencia. Recuperado de http:// www.transparencia.org.pe/admin//ckfinder/ userfiles/files/00\%20REPORTE\%201a\%20 VUELTA.pdf

Chávez Salazar, R. (1999). El derecho de petición y la Administración Pública en el Perú. Themis(39), pp. 189-203. Recuperado el 19 de febrero de 2018 de http://revistas.pucp.edu.pe/ index.php/themis/search/authors/view?firstNa me $=$ Ricardo\&middleName $=\&$ lastName $=$ Sala zar\% $20 \mathrm{Ch} \% \mathrm{C} 3 \% \mathrm{~A} 1$ vez\&affiliation=Pontific ia $\% 20$ Universidad $\% 20$ Cat $\%$ C3\%B3lica $\% 20$ del $\% 20$ Per $\%$ C $3 \%$ BA\&country $=$ PE

Hernández Godínez, A. (2010). El acceso a la información pública: evolución y consolidación de un derecho fundamental de nueva generación. Getafe, Madrid, España: Universidad Carlos III Madrid. Recuperado el 20 de febrero de 2018 de http://hdl.handle. net/10016/8335

Latinobarómetro. (2016). Libro de códigos Perú. Corporación Latinobarómetro. Santiago de Chile: Latinobarómetro. Recuperado el 22 de febrero de 2018 de http://www. latinobarometro.org/latCodebooks.jsp

Ministerio de Justicia y Derechos Humanos. (2014). Guía práctica sobre la transparencia y el acceso a la información pública. Lima, Perú : Dirección General de Desarrollo y
Ordenamiento Jurídico. Recuperado el 27 de febrero de 2018 de https://www.minjus.gob. pe/wp-content/uploads/.../DGDOJ-Guía-deTransparencia.pdf

Palomares Herrera, M. (2017). Estado de la transparencia y el emergente derecho fundamental de acceso a la información pública en España. Jaén, España: Universidad de Jaén - Facultad de Ciencias Sociales y Jurídicas. Recuperado el 20 de febrero de 2018 de http://ruja.ujaen.es/ bitstream/10953/860/3/9788491590927.pdf

Precht, A. y Figueroa, M. (3 de enero - abril de 2017). Transparencia en los partidos políticos: el modelo chileno. Revista internacional Transparencia e Integridad (3), pp. 1 -10. Recuperado el 20 de febrero de 2018 de https://revistainternacionaltransparencia.org/ wp-content/uploads/2017/05/Alberto-Brechty-Michel-Figueroa.pdf

Rubio Correa, M. (1999). Estudio de la Constitución Política de 1993 (1. a edición, vol. I). Lima, Perú: Pontificia Universidad Católica del Perú. Recuperado el 20 de febrero de 2018 de http://repositorio.pucp.edu.pe/index/ handle/123456789/68

Santana Sabbagh, C. (2014). El acceso a la información pública como derecho fundamental. Sevilla, Sevilla, España: Universidad de Sevilla. Recuperado el 21 de febrero de 2018 de http://masteroficial.us.es/ derecho/sites/masteroficial.us.es.derecho/files/ TFM_Carolina_Santana.pdf

Tribunal Constitucional, Expediente 026292012-AA (Tribunal Constitucional 22 de octubre de 2012). Recuperado el 20 de febrero de 2018 de http://www.tc.gob.pe/ jurisprudencia/2012/02629-2012-AA.html

Tuesta Soldevilla, F. (2008). Regulación jurídica de los partidos políticos en Perú. En Zovattoy Zovatto (Ed.), Regulación Jurídica de los Partidos Políticos en América Latina (pp. 767-802). México D.F, México: Universidad Autónoma de México - IDEA Internacional. Recuperado el 20 de febrero de 2018 de http://blog.pucp. edu.pe/blog/fernandotuesta/wp-content/ uploads/sites/945/2016/05/C-2008-UNAM.Regulacion-Juridica-de-los-PartidosPoliticos-en-America-Latina.pdf 
Tuesta Soldevilla, F. (2012). Nacionales, Fortalezas y Desafíos en la Regulación del Financiamiento Político y de las Campañas Electorales en América Latina. Apreciación de Expertos. En The Carter Center, Financiamiento Político y regulación de camapañas electorales en América Latina (págs. 28 - 30). Atlanta: The Carter Center. Recuperado el 20 de Febrero de 2018, de https://www.cartercenter.org/resources/pdfs/ news/peace_publications/americas/electoral_ code_booklet-vdg_a2_final2.pdf

Valverde Loya, M. Á. (5 de diciembre de 2007). Transparencia, acceso a la información y rendición de cuentas. Revista Jurípolis(7), pp. 235- 250. de lhttp://catedraunescodh. unam.mx/catedra/CONACYT/04_Docentes UdeO_ubicar_el_de_alumnos/Contenidos/ Lecturas\%20obligatorias/M.5_cont_1 Valverde_Loya.pdf

Ley $\mathrm{N}^{\circ}$ 28094, Ley de organizaciones Políticas (01 de noviembre del 2003), Recuperado del sitio de internet del jurado nacional de elecciones: http://aplicaciones007.jne.gob.pe/srop/ documentos/DocLegales/LeyOP28094_.pdf

Ley $\mathrm{N}^{\circ} 27806$, Ley de Transparencia y Acceso a la Información Pública (03 agosto del 2002), Recuperada del sitio de internet del Congreso de la Republica: http://www.leyes.congreso. gob.pe/Documentos/Leyes/27806.pdf

\section{BIBLIOGRAFÍA COMPLEMENTARIA}

Mujica, J. (2014). El lobby en un escenario de agendas fragmentadas. Consideraciones sobre los mecanismos de gestión de intereses en el Parlamento peruano. Revista de Ciencia Política y Gobierno, 1(1), pp. 3754. Recuperado el 20 defebrero de 2018 de http://revistas.pucp.edu.pe/index.php/ cienciapolitica/article/view/11891/12460

Poder Judicial. (2015). Quinto Pleno Casatorio Civil. Lima, Perú : Fondo Editorial del Poder Judicial.

Tanaka, M.; Vera, S. y Barrenechea, R. (2009). Think tanks y partidos políticos en el Perú: precariedad institucional y redes informales. En Mendizabal y Sample (Edits.), Dime a quién escuchas... think tanks y partidos políticos en América Latina. (pp. 209 - 239). Lima, Perú: IDEA Internacional - Overseas Development Institute. Recuperado el 19 de febrero de 2018 de https://www.idea.int/publications/ catalogue/thinking-politics-think-tanks-andpolitical-parties-latin-america?lang=es

Transparencia Internacional España. (2017). Evaluación del nivel de transparencia de los Partidos políticos. Madrid: Transparencia Internacional España. Recuperado de https://transparencia.org.es/wp-content/ uploads/2017/06/evaluacion_nivel_transp_ partidos-junio-2017.pdf 